\title{
Estudio del acoplamiento de diferentes configuraciones de ciclo combinado con planta solar de canal parabólico
}

\section{Study of Different Configurations of ISCC Parabolic Through}

\author{
Durán-García María Dolores \\ Facultad de Ingeniería \\ Universidad Autónoma del Estado de México \\ Correo:mddg_2210@hotmail.com \\ Almanza-Salgado Rafael \\ Instituto de Ingeniería \\ Universidad Nacional Autónoma de México \\ Correo:ras@pumas.iingen.unam.mx
}

\author{
Martínez-Cienfuegos Ivan Galileo \\ Facultad de Ingeniería \\ Universidad Autónoma del Estado de México \\ Correo:igmartinez@uaemex.mx
}

Información del artículo: recibido: diciembre de 2013, reevaluado: enero y marzo 2014, aceptado: abril 2014

\section{Resumen}

Este trabajo presenta el estudio del acoplamiento de una planta solar con ciclo combinado. Se estudian diferentes configuraciones considerando la planta solar como el economizador o el sobrecalentador de la caldera de recuperación de calor. El objetivo es obtener el diseño óptimo, desde el punto de vista termoeconómico de la caldera, incluyendo la planta solar, determinando los parámetros de diseño optimizados para ambos sistemas. Se aplica una metodología empleada en trabajos previos para la optimización de ciclos combinados, pero incluyendo ahora la planta solar. Asimismo, se realiza un análisis de sensibilidad con respecto a la variación de radiación solar, tomando un día promedio del mes de mayo. Como resultado se obtiene el rendimiento y costo óptimos de las configuraciones analizadas.

\footnotetext{
Abstract

This paper presents the study of the coupling of a solar plant with a combined cycle. Different configurations are analyzed considering the solar plant as economizer or superheated boiler with heat recovery. The goal is to obtain the thermoeconomic optimal design of the boiler, including the solar plant, so determining the optimized design parameters for both systems. The methodology used in previous papers to optimize the combined cycle was used, but now including the solar plant. Also, a sensitivity analysis with respect to the variation of solar radiation is conducted, taking the average day of May. As a result, the best performance and cost of the obtained configurations is analyzed.
}

\section{Descriptores:}

- planta de canal parabólico

- planta solar integrada a un ciclo combinado

- análisis termoeconómico

\section{Keywords:}

- integrated solar combined cycle plant

- parabolic trough plant

- thermoeconomic analysis 


\section{Introducción}

La electricidad generada a partir de energía solar-térmica (Solar Thermal Electricity, STE) es una tecnología probada con más de 30 años de experiencia esperando que sea competitiva con las centrales térmicas de combustible fósil para el 2020 (Llorente et al., 2011; IEA, 2011). Esta tecnología está disponible principalmente a partir de plantas tipo CSP (Concentrating Solar Power) instaladas en regiones áridas o semi-áridas.

Actualmente existen cuatro tecnologías comerciales disponibles de sistemas de concentración solar (CSP); dos de ellas, el canal parabólico y el concentrador Fresnel linear reflejan la radiación solar hacia un concentrador lineal, mientras que las otras dos la concentran en un punto.

A finales de 2010, había 1318 MW de capacidad instalada acumulada de CSP en todo el mundo. Hasta ese año, España fue el líder mundial en instalaciones termosolares, con $450 \mathrm{MW}$ de capacidad adicional, acumulando $55.4 \%$ de la capacidad instalada en todo el mundo. Mientras tanto, Estados Unidos añadió $78 \mathrm{MW}$ de potencia CSP, para un total de $38.5 \%$ de la capacidad acumulada mundial, mientras que otros países como: Irán (5.0\%), Israel (0.5\%), Australia (0.2\%) y Alemania $(0.1 \%)$ juntos sumaron un porcentaje menor (U.S. DE, 2011).

La tecnología CSP del tipo canal parabólico es la única que ha demostrado su funcionamiento comercial a largo plazo, esto se debe a su corto tiempo de puesta en marcha y gran tiempo de operación (más de 30 años). Actualmente existen alrededor de 29 plantas en operación y más de 1220 MWe instalados, lo que corresponde a 96\% del total de sistemas CSP instalados (Lorente et al., 2011).

Así pues, el potencial de los CSP, principalmente de canal parabólico y torre central, es muy grande y se tiene la expectativa de que su capacidad instalada se incremente considerablemente en los próximos años, debido a la disminución de precios. De acuerdo con IEA-ETSAP e IRENA (2013), se espera que para 2015, derivado de los desarrollos tecnológicos y la economía de escala, el costo de generación con este tipo de tecnologías disminuya $15 \%$, y para el 2020 podría disminuir entre 30 y $50 \%$. Este mismo reporte sugiere que el éxito de los sistemas CSP se verá sumamente apoyado con la hibridación de estos con plantas térmicas que emplean combustibles fósiles, particularmente ciclo combinado con turbina de gas y de vapor (conocidos como ISCC), como el que se presenta en este trabajo. Por su parte, el GEF (2006), en su programa operacional incluye la demostración de la viabilidad de estos sistemas con la in- stalación de una planta con 31MW solares y 530MW de ciclo combinado en la región de Agua Prieta, Sonora, México.

Con respecto a la hibridación de sistemas con plantas térmicas de combustibles fósiles, se realizaron diversasinvestigaciones(Monteset al., 2011;Nezammahalleh et al., 2010) sobre la combinación de plantas solares con ciclo combinado (ISCC), las cuales emplean la energía solar como energía suplementaria a la que proviene de la turbina de gas; esto permite un incremento de la potencia generada por la turbina de vapor, y una compensación de la disminución del rendimiento de la turbina de gas bajo ciertas condiciones ambientales (Valdés et al., 2006a). En la mayoría de las configuraciones propuestas el campo de canal parabólico se puede utilizar como el economizador de la caldera de recuperación de calor (Montes, 2008).

Tomando los trabajos mencionados como precedente, el objetivo de este artículo es presentar un análisis de diferentes configuraciones de plantas de canal parabólico con generación directa de vapor (GDV) integradas con ciclo combinado.

Ahora bien, se propone la generación directa de vapor porque se considerará al campo solar como el economizador o el sobrecalentador de la planta, por lo que no habrá flujo bifásico en el absorbedor del concentrador. Además, existen varios trabajos que han estudiado la generación directa de vapor (Lentz y Almanza, 2006; Zarza, 2006), que muestran que esta es factible tanto a baja como a alta presión. También está el de Montes (2008), en el cual se compara el desempeño de una planta solar GDV con aquella que utiliza aceite Therminol VP-1 como fluido de trabajo, concluyendo que la GDV presenta un mayor rendimiento energético y exergético porque no requiere un intercambiador de calor.

En cuanto al análisis térmico de la planta solar, distintos trabajos lo realizan, como el de Bakos et al. (2001), que muestran la variación del rendimiento del colector parabólico como función del fluido de transferencia de calor. Asimismo, el de Tyagi et al. (2007) que está relacionado con el análisis por segunda ley de este tipo de sistemas. Sin embargo, existen pocos trabajos relacionados con el análisis termoeconómico y la optimización de los mismos (Baghernejad y Yaghoubi, 2011). Por esta razón se eligió realizar este análisis, considerando además que en trabajos previos (Valdés et al., 2003; Durán et al., 2013) se ha realizado este tipo de análisis a otros sistemas y se han obtenido resultados más realistas.

En trabajos previos realizados por los autores (Durán, 2004), se desarrolló un modelo de optimización termoeconómica con algoritmos genéticos que se aplicó a plantas de ciclo combinado (CC), por lo que se propone 
aplicar este mismo modelo a la planta de ciclo combinado, integrada con la planta solar.

\section{Desarrollo}

Descripción y parámetros de la planta solar integrada con ciclo combinado

Descripción: en este trabajo se realiza el análisis de diferentes configuraciones de plantas de canal parabólico integradas con un ciclo combinado (ISCC, Integrated Systems with Combined Cycle). Estas plantas constan de un ciclo combinado común pero incluyen una planta solar que sustituye a una sección de la caldera, consta de los siguientes elementos:

Turbina de gas: transforma la energía térmica de los gases que ingresan a alta temperatura y alta presión provenientes de la cámara de combustión en trabajo. Los gases de escape de este elemento aún tienen suficiente entalpía que puede ser aprovechada en un ciclo de baja temperatura (ciclo de vapor).

Caldera de recuperación de calor: es el elemento de unión entre el ciclo de gas y de vapor, es el encargado de recuperar la energía térmica de los gases provenientes de la turbina de gas para producir vapor. Consta de tres secciones por cada nivel de presión:

- Economizador (EC). En esta sección el agua se calienta hasta unos grados (Approach Point) menos que el punto de saturación a la presión de trabajo de la caldera. Para CRC de dos niveles de presión se suele tener uno o dos economizadores por cada nivel de presión.

- Evaporador (EV). En esta sección de la caldera presenta el cambio de fase y se lleva al agua desde líquido saturado hasta vapor saturado a la presión nominal. Se suele tener un evaporador por cada nivel de presión de la CRC.

- Sobrecalentador (EV). En esta última sección se lleva el vapor saturado a sobrecalentado. Se suele tener un sobrecalentador por cada nivel de presión de la CRC.
En el presente trabajo el campo solar sustituirá, según el caso, al economizador o al sobrecalentador de un nivel de presión de la caldera.

Planta solar. Se trata de una planta de canal parabólico, con generación directa de vapor (GDV) en el presente trabajo sustituirá alguna sección de la caldera de recuperación de calor. La planta solar está constituida por un número lazos de concentradores solares conectados en paralelo, cada lazo es un conjunto de concentradores solares en serie.

Turbina de vapor. Es el elemento de potencia del ciclo de baja temperatura, recibe al vapor de la CRC y lo transforma en trabajo adicional.

En el presente trabajo se estudian 3 configuraciones con 2 niveles de presión sin recalentamiento y sin almacenamiento térmico en el campo solar:

- La configuración de ciclo combinado sin planta solar.

- El campo solar es parte del economizador de alta presión (figura 1).

- El campo solar es parte del sobrecalentador de baja presión (figura 2).

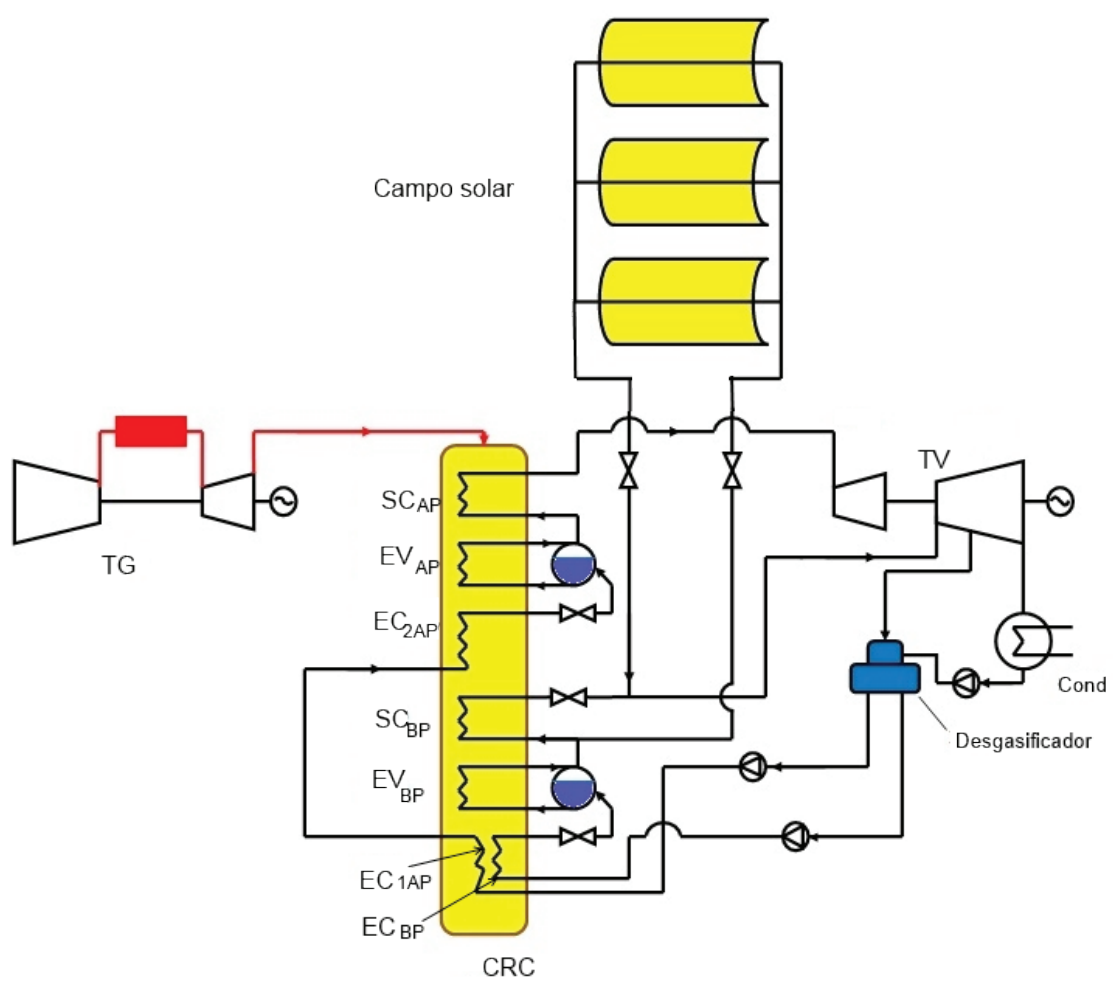

Figura 1. Configuración B: Planta de dos niveles de presión sin recalentamiento, con el campo solar acoplado al economizador de alta 


\section{Parámetros de diseño y consideraciones}

Para el análisis que aquí se presenta los parámetros de diseño que se consideran durante la optimización corresponden al ciclo de vapor, particularmente a la CRC y a la planta solar. Los parámetros de diseño más importantes de la caldera son los siguientes:

- Presión nominal. Es la presión de operación de cada nivel de presión de la caldera.

- Pinch Point (PP). Corresponde a la diferencia de temperaturas entre el agua líquida saturada a la entrada del evaporador y los productos de la combustión a la salida del mismo. Este valor en gran medida determinará el tamaño de la caldera y por lo tanto del campo solar. Si se diseñara una caldera únicamente considerando la optimización térmica, este valor tendería a ser muy pequeño, sin embargo, esto ocasionaría un incremento sumamente importante en el costo de la caldera de recuperación de calor o del campo solar en su caso.

- Approach Point (AP). Corresponde a la diferencia de temperaturas entre el agua que sale del economizador y la temperatura de saturación de la misma a la presión nominal. Este valor es un parámetro de seguridad que permitirá evitar evaporación en el economizador y por lo tanto en el campo solar cuando sustituya a esta sección de la caldera.

- Diferencia de temperaturas en el sobrecalentador. Corresponde a la diferencia de temperaturas entre el vapor a la salida del sobrecalentador y los gases a

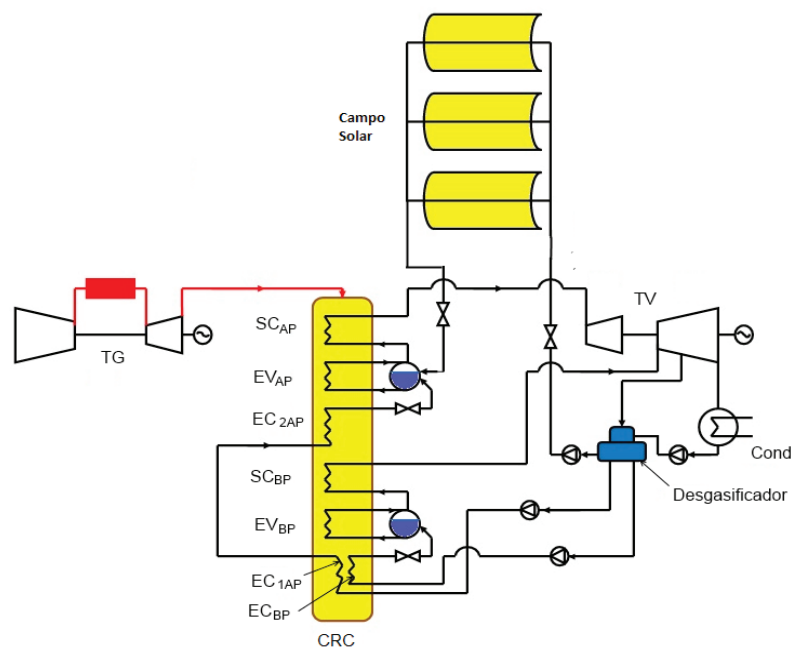

Figura 2. Configuración C: Planta de dos niveles de presión sin recalentamiento con el campo solar acoplado al sobrecalentador de baja la entrada del mismo. Esta diferencia determina el tamaño del sobrecalentador y del campo solar si este está acoplado en esta sección.

Ahora bien, en cuanto a los parámetros de diseño de la turbina de gas y de vapor, estos no se considerarán como variables independientes en el proceso de optimización. Se elige una turbina de gas comercial cuyas características de diseño se presentan en la tabla 1. Por su parte, los parámetros relacionados con la simulación del ciclo de vapor tampoco se incluirán dentro de la optimización, se empleará un valor fijo, el cual se presenta en la tabla 2. En el presente trabajo se describe la optimización del ciclo centrada en los parámetros de diseño de la CRC y el campo solar; esta optimización se realizará aplicando la herramienta de algoritmos genéticos, para lo cual debe considerarse un intervalo de variación de los parámetros de diseño (tabla 3). Se puede observar en esta tabla que se proponen valores límite del pinch point y approach point entre 3 y $15 \mathrm{~K}$. Se propone esto porque es lo sugerido para centrales de ciclo combinado y calderas de recuperación de calor (Horlock, 1991).

La optimización del sistema se realiza considerando una irradiancia directa promedio mensual correspondiente a la zona de Cerro Prieto, en Baja California, México, de acuerdo con datos de Google ${ }^{\mathrm{TM}}$ Earth, la ubicación geográfica del lugar tiene una latitud de $32.40^{\circ} \mathrm{N}$ y una longitud de $115.24^{\circ} \mathrm{O}$. Las condiciones de Cerro Prieto se presentan en la tabla 4.

Tabla 1. Parámetros de diseño del ciclo de gas

\begin{tabular}{ll}
\hline Parámetro & Valor \\
\hline Relación de compresión & 30 \\
Pérdida de carga en la admisión & $20 \mathrm{mbar}$ \\
Rendimiento isentrópico del compresor & 0.85 \\
Rendimiento de la cámara de combustión & 0.95 \\
Rendimiento isentrópico de la turbina & 0.91 \\
Temperatura de entrada a la turbina & $1430 \mathrm{~K}$ \\
Temperatura de salida & $710 \mathrm{~K}$ \\
Flujo másico de aire & $120.2 \mathrm{~kg} / \mathrm{s}$ \\
Pérdida de carga en el escape & $40 \mathrm{mbar}$ \\
\hline Resultados de la simulación & \\
\hline Potencia nominal de la turbina de gas & $38.8 \mathrm{MWe}$ \\
Rendimiento del ciclo de gas & $40.12 \%$ \\
Temperatura de entrada a la CRC & $710.7 \mathrm{~K}$ \\
Temperatura de la mezcla & $1222.19 \mathrm{~K}$ \\
\hline
\end{tabular}


Tabla 2. Parámetros de diseño de la turbina de vapor

\begin{tabular}{ll}
\hline Parámetro & Valor \\
\hline Número de niveles de presión & 2 \\
$\begin{array}{l}\text { Rendimiento isentrópico de la turbina de } \\
\text { vapor }\end{array}$ & 0.85 \\
$\begin{array}{l}\text { Presión del condensador } \\
\text { Presión del desgasificador }\end{array}$ & $0.045 \mathrm{bar}$ \\
\hline
\end{tabular}

Tabla 3. Parámetros para la optimización termoeconómica con su intervalo de variación en el algoritmo

\begin{tabular}{ll}
\hline Parámetro de diseño & $\begin{array}{l}\text { Intervalo de } \\
\text { variación }\end{array}$ \\
\hline Presión en colector de baja & $3-15 \mathrm{bar}$ \\
Pinch Point de la sección de baja presión & $3-15 \mathrm{~K}$ \\
$\begin{array}{l}\text { Approach Point de la sección de baja } \\
\text { presión }\end{array}$ & $3-15 \mathrm{~K}$ \\
$\begin{array}{l}\text { Diferencia de temperatura en el } \\
\text { sobrecalentador de baja presión }\end{array}$ & $20-70 \mathrm{~K}$ \\
$\begin{array}{l}\text { Presión en el colector de alta } \\
\text { Pinch Point de la sección de alta presión }\end{array}$ & $30-100 \mathrm{bar}$ \\
$\begin{array}{l}\text { Approach Point de la sección de alta } \\
\text { presión }\end{array}$ & $3-15 \mathrm{~K}$ \\
$\begin{array}{l}\text { Diferencia de temperatura en el } \\
\text { sobrecalentador de alta presión }\end{array}$ & $20-80 \mathrm{~K}$ \\
\hline
\end{tabular}

Tabla 4. Datos geográficos y ambientales de Cerro Prieto, Baja California

\begin{tabular}{ll}
\hline Parámetro & Valor \\
\hline Latitud & $32,40^{\circ} \mathrm{N}$ \\
Longitud & $115,24^{\circ} \mathrm{O}$ \\
Altitud & $12 \mathrm{msnm}$ \\
$\begin{array}{l}\text { Temperatura ambiente máxima normal } \\
\text { promedio anual }\end{array}$ & $32,2^{\circ} \mathrm{C}$ \\
Irradiación directa normal diaria promedio & $23,79 \mathrm{MJ} / \mathrm{m} 2$ \\
mensual (Almanza y Estrada, 2005). & \\
& \\
Irradiancia directa instantánea & $797 \mathrm{~W} / \mathrm{m} 2$ \\
\hline
\end{tabular}

\section{Análisis termodinámico y termoeconómico}

Análisis termodinámico de la planta solar

El rendimiento térmico $(\eta)$ se define como la razón de la ganancia térmica útil $\left(Q_{u}\right)$ en un periodo de tiempo $(d t)$, entre la energía solar incidente (integral de la irradiancia global, $\mathrm{G}_{\mathrm{T}}$ ) multiplicada por el área de apertura del concentrador $\left(A_{A}\right)$ en el mismo intervalo de tiempo. $\eta=\frac{\int Q_{u} d t}{A_{A} \int G_{T} d t}$

El periodo de tiempo típico es de $1 \mathrm{~h}$, pero si se tienen datos de irradiación global $\left(I_{T}\right)$ de un día, entonces el periodo de tiempo serán las horas pico del día en promedio para un mes o para un año.

Asimismo se define otra variable que relaciona la ganancia útil de energía instantánea de un concentrador entre la ganancia útil si toda la superficie del absorbedor estuviera a la temperatura del fluido, a esto se le llama factor de remoción de calor del concentrador $\left(\mathrm{F}_{\mathrm{R}}\right)$.

El modelo termodinámico aplicado determina el rendimiento térmico instantáneo $\left(\eta_{i}\right)$ del colector parabólico en función del factor de remoción de calor (Duffie y Beckman, 2006), en el que se toma en cuenta la irradiancia directa instantánea $\left(G_{b}\right)$

$\eta_{i}=\frac{Q_{u}}{A_{A} G_{b}}=\frac{F_{R}\left[G_{b}(\tau \alpha)-U_{L}\left(T_{i}-T_{a}\right)\right]}{G_{b} C}$

donde

$\mathrm{U}_{\mathrm{L}}=$ coeficiente global de transferencia de calor

$\mathrm{C}=$ razón de concentración solar

$\tau=$ transmitancia

$\alpha=$ absortancia del material del absorbedor

$T_{i}=$ temperatura de entrada del fluido al colector

$T_{a}=$ temperatura ambiente

En esta ecuación el factor de extracción de calor $\left(\mathrm{F}_{\mathrm{R}}\right) \mathrm{se}$ determina mediante la ecuación

$$
F_{R}=\frac{\dot{m} C_{P}}{A_{A} U_{L}}\left[1-\exp \left(-\frac{A_{A} U_{L} F^{\prime}}{\dot{m} C_{P}}\right)\right]
$$

Donde el factor de eficiencia del colector $\left(\mathrm{F}^{\prime}\right)$ está dado como la razón de la resistencia de transferencia de calor del absorbedor y el aire ambiente $\left(\mathrm{U}_{\mathrm{o}}\right)$ entre la resistencia de transferencia de calor del fluido de trabajo y el ambiente $\left(\mathrm{U}_{\mathrm{L}}\right)$, esto significa que la mejora en el rendimiento de captación de la energía en el concentrador es función del coeficiente global de transferencia de calor y del factor transferencia de calor por convección del fluido. Por su parte $\dot{m}$ es el flujo másico $\left(C_{p}\right)$ es el calor específico a presión constante y $A_{A}$ es el área de apertura total del concentrador (Tiwari, 2002).

El rendimiento del colector determinado mediante la ecuación (2) para un periodo diario promedio anual, se empleará para obtener el tamaño de la planta solar en el proceso de optimización. 


\section{Análisis termodinámico del ciclo combinado}

Se aplicó un programa desarrollado en macros de Visual Basic para Excel para simular y optimizar el ciclo combinado integrado con el campo de canal parabólico. Este programa emplea el modelo de costo-flujo de caja, propuesto por Rovira (2004).

En el método aplicado se realiza la simulación del ciclo de gas operando en condiciones de diseño, aplicando el modelo descrito en Muñoz (2004) y Facchinni y Stecco (1999). La caldera de recuperación de calor, el campo solar y el ciclo de vapor se simulan aplicando las correlaciones de la IAPSW (Wagner y Kretzchmar, 1988).

\section{Modelo económico}

La optimización del sistema se realiza minimizando el costo de generación, el cual corresponde al costo total anual dividido por la energía generada anualmente, es decir

$$
C_{k W h}=\frac{C_{T o t}}{\bar{W} \cdot h}
$$

En la ecuación (3), el costo total anual $\left(\mathrm{C}_{\mathrm{Tot}}\right)$ incluye el costo de amortización, el costo del combustible y el de operación y mantenimiento de todo el sistema, considerando al campo solar. Por su parte, $\bar{W}$ es la potencia media anual de la planta y $h$ es el número de horas de operación del sistema por año. En el presente trabajo se consideraron un total de 7000 horas para la planta de ciclo combinado y 1460 para el campo solar.

Para llevar a cabo el análisis económico del ciclo combinado se consideraron las funciones de costo de la turbina de gas, turbina de vapor y caldera de recuperación de calor que se describen en Durán (2004).

- Costo de la turbina de gas: este costo se determinó del análisis de tendencias del costo de turbinas de gas (Valdés et al., 2006b), en el cual se obtiene una expresión del costo de la turbina de gas en función de la potencia de la misma, expresada en MW.

-

$$
C_{T G}=0.1788 W_{T G}+3.0253
$$

- Costo de la turbina de vapor: este costo se determinó del análisis de tendencias del costo de turbinas de vapor (Valdés et al., 2006b), en el cual se obtiene una expresión del costo de la turbina de vapor en función de la potencia de la misma, expresada en MW.

$$
C_{T V}=0.115 W_{T V}+2.75
$$

- Costo de la CRC: incluye varios componentes como el costo del diseño, de la estructura e instalación y el costo de los materiales de la superficie de intercambio de calor. Sería deseable conocer todos estos costos, sin embargo, se cuenta con muy poca información al respecto. Es necesario desarrollar un modelo que sea capaz de predecir el costo de dicha caldera en función de sus parámetros termodinámicos, sin conocer el diseño geométrico de la misma. En la bibliografía se encuentran varios modelos que desarrollan correlaciones para predecir este costo. Algunos autores (Attala et al., 2001) y (Valero, et al. 1994) definen el costo de cada sección de la caldera en función del producto UA de la forma:

$$
C_{f-C R C, \text { sec }}^{U A}=\sum_{\mathrm{sec}} k_{\mathrm{sec}}(U A)_{\mathrm{sec}}^{x}
$$

donde el exponente $(x)$ al que se encuentra elevado el producto $U A$ se incluye debido a la economía de escala, es decir, que cuanto mayor sea el área de la caldera, más barata será la unidad de UA. El valor empleado en este trabajo es el propuesto por Valero et al. (1994) y Tsatsaronis y Pisa (1994) quienes consideran este exponente como 0.8 y proponen el costo de la caldera de la siguiente forma

$$
C_{f-C R C, \text { sec }}^{U A}=\sum_{\text {sec }}^{n s e c} k_{\text {sec }}(U A)_{\mathrm{sec}}^{0.8}
$$

Por otra parte, para realizar el análisis económico de la planta solar se tomaron en cuenta los costos establecidos por Montes et al. (2011). Vea la tabla 5.

En cuanto a los parámetros financieros, se tomó una tasa de interés de $10 \%$ y un tiempo de operación de 25 años, se consideró este valor porque es el tiempo promedio de operación de una planta de ciclo combinado.

El método de optimización que se aplicó es el de algoritmos genéticos (AG), ya que este se aplicó en trabajos previos relacionados con la optimización de ciclos combinados, donde presentaron resultados bastante aceptables. El algoritmo de optimización se explica me-

Tabla 5. Consideraciones para el análisis económico del campo solar

\begin{tabular}{ll}
\hline Costo de inversión del campo solar $\left(€ / \mathrm{m}^{2}\right)$ & 200 \\
Costo del terreno $\left(€ / \mathrm{m}^{2}\right)$ & 2 \\
$\begin{array}{l}\text { Costo de operación y mantenimiento de la } \\
\text { planta solar }\left(€ / \mathrm{m}^{2}\right)\end{array}$ & 9 \\
\hline
\end{tabular}


diante el diagrama de la figura 3. Como se indica, para cada generación el AG genera aleatoriamente un número establecido de individuos que incluyen las variables de optimización, con ellas se simula el ciclo y la planta solar y se calcula el costo de generación para las condiciones dadas. Finalmente se selecciona la combinación de variables que proporcione el menor costo de generación. Mayor información de esta metodología y su aplicación en la optimización de sistemas térmicos puede encontrarse en Toffolo y Lazzareto (2002) y Valdés et al. (2003).

\section{Discusión y análisis de resultados}

\section{Parámetros considerados para la simulación de la planta solar}

Con el fin de realizar la evaluación numérica de los resultados, se consideró un captador parabólico comercial modelo Eurotrough ET-100. Las especificaciones técnicas de este tipo de captador se presentan en la tabla 6. Especificaciones más detalladas de este tipo de colectores, así como un análisis de su desempeño se puede encontrar en Geyer et al. (2002).

Es importante señalar que se consideró un flujo másico dentro del absorbedor de $1.4 \mathrm{~kg} / \mathrm{s}$ porque es el valor sugerido para evitar grandes pérdidas de carga en el mismo (Montes, 2009).

\section{Resultados de la optimización}

Tomando en cuenta las consideraciones mencionadas en los apartados anteriores, se aplicó el programa de optimización a las configuraciones analizadas. Los resultados de la optimización se obtuvieron considerando el mínimo costo de generación (tabla 7).

Tabla 6. Parámetros de la planta de canal parabólico

\begin{tabular}{ll}
\hline \multicolumn{2}{c}{ Parámetros del modulo solar } \\
\hline Modelo del colector solar & ET100 \\
Radio del absorbedor & $0.035 \mathrm{~m}$ \\
Área de la superficie reflejante & $545 \mathrm{~m}^{2}$ \\
Apertura & $5.77 \mathrm{~m}$ \\
Longitud del colector & $99.5 \mathrm{~m}$ \\
Reflectividad del espejo & $94 \%$ \\
Rendimiento óptico pico & $80 \%$ \\
Razón de concentración & 23.23 \\
Longitud de cada modulo & $12 \mathrm{~m}$ \\
Flujo másico en el absorbedor & $1.4 \mathrm{~kg} / \mathrm{s}$ \\
\hline
\end{tabular}

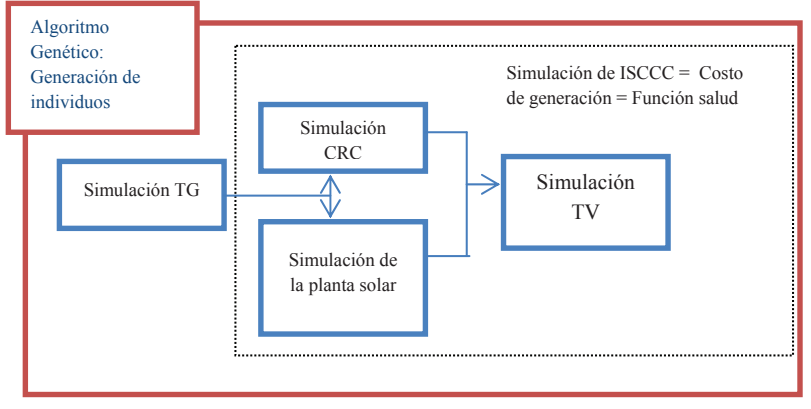

Figura 3. Diagrama de flujo del programa de optimización

Es importante aclarar que se trata de una optimización termoeconómica, si se tratara solo de una optimización térmica podría esperarse que los valores del pinch point y el approach point tendieran al mínimo del intervalo, sin embargo, al involucrar el costo el programa de optimización encuentra el valor óptimo con el que se obtiene el menor costo de generación. Por esta razón los valores de la tabla 7 no corresponden al límite inferior del intervalo propuesto.

Primero se optimizó la planta de ciclo combinado de dos niveles de presión, sin recalentamiento y sin el acoplamiento del campo solar, estos resultados se muestran en la segunda columna de la tabla 7. Se observa que esta configuración presenta un rendimiento de $54.68 \%$ y un costo de generación de $0.529 € / \mathrm{kWh}$. Estos valores se encuentran en las tendencias de diseño de estos sistemas (Valdés et al., 2006b).

En la columna 3 se presenta la optimización de la ISCC, considerando al campo solar como parte del economizador de alta presión. Se observa que esta configuración es la que presenta un menor costo de generación, ya que el acoplamiento del campo solar en la sección de alta presión deja más energía disponible de los gases calientes en la CRC en la zona de baja presión de la misma, permitiendo incrementar el flujo másico en esta última sección y aumentando así la potencia en la turbina de baja.

Por su parte, en la configuración donde el campo solar es el sobrecalentador de la sección de baja presión de la CRC (Configuración C, ISCC), se observa que, si bien, el rendimiento es mayor que en los otros dos casos, el costo de generación es mayor que el obtenido cuando el campo solar está acoplado en el economizador de alta presión, aunque menor que el obtenido con la configuración A. Esto se debe a que el acoplamiento con el campo solar sí tiene un efecto en el incremento del flujo másico de vapor del ciclo de baja, pero es mucho menor porque la energía que se transfiere en este sobrecalentador es mucho menor. 
Tabla 7. Resultados de la optimización para las configuraciones analizadas

\begin{tabular}{|c|c|c|c|}
\hline Parámetros termodinámicos & $\begin{array}{c}\text { Config. } \\
\text { A CCPP } \\
\text { s/canal } \\
\text { parabólico } \\
\text { optimizada }\end{array}$ & $\begin{array}{l}\text { Config. B } \\
\text { ISCC }\end{array}$ & $\begin{array}{c}\text { Config. C } \\
\text { ISCC }\end{array}$ \\
\hline Presión de baja & $324 \mathrm{kPa}$ & $389 \mathrm{kPa}$ & $371 \mathrm{kPa}$ \\
\hline PP de baja & $3.01 \mathrm{~K}$ & $14.5 \mathrm{~K}$ & $9.4 \mathrm{k}$ \\
\hline AP de baja & $4.07 \mathrm{~K}$ & $5.91 \mathrm{~K}$ & $3.09 \mathrm{~K}$ \\
\hline DT de baja & $84.8 \mathrm{~K}$ & $69.24 \mathrm{~K}$ & $51.55 \mathrm{~K}$ \\
\hline Presión de alta & $6621 \mathrm{kPa}$ & $9639 \mathrm{kPa}$ & $10168 \mathrm{kPa}$ \\
\hline PP de alta & $3.98 \mathrm{~K}$ & $12.455 \mathrm{~K}$ & $14.83 \mathrm{~K}$ \\
\hline AP de alta & $7.6 \mathrm{~K}$ & $4.25 \mathrm{~K}$ & $7.59 \mathrm{~K}$ \\
\hline DT de alta & $43.145 \mathrm{~K}$ & $69.95 \mathrm{~K}$ & $50.5 \mathrm{~K}$ \\
\hline Costo/kWh & $\begin{array}{l}0.05298 € / \\
\text { kWh }\end{array}$ & $\begin{array}{l}0.04602 € / \\
\text { kWh }\end{array}$ & $\begin{array}{l}0.052 € / \\
\mathrm{kWh}\end{array}$ \\
\hline Rendimiento & $54.68 \%$ & $56.0 \%$ & $57.9 \%$ \\
\hline Potencia del CC & $52986.82 \mathrm{~kW}$ & $54286.33 \mathrm{~kW}$ & $53083.0 \mathrm{~kW}$ \\
\hline Flujo másico de alta presión & $10.89 \mathrm{~kg} / \mathrm{s}$ & $10.95 \mathrm{~kg} / \mathrm{s}$ & $9.26 \mathrm{~kg} / \mathrm{s}$ \\
\hline Flujo másico de baja presión & $5.32 \mathrm{~kg} / \mathrm{s}$ & $9.20 \mathrm{~kg} / \mathrm{s}$ & $5.84 \mathrm{~kg} / \mathrm{s}$ \\
\hline Número de bucles totales & 0 & 26 & 9 \\
\hline $\begin{array}{l}\text { Número de bucles en } \\
\text { paralelo }\end{array}$ & 0 & 7 & 7 \\
\hline
\end{tabular}

entonces durante este tiempo se tiene un incremento considerable en el flujo de vapor de alta y por lo tanto en el rendimiento del ciclo. Durante las horas de menor irradiancia la falta de energía solar se compensa con la de la turbina de gas, debido a que el vapor que no genera el campo solar, lo genera la CRC en su sección del economizador de alta presión; esto implica una disminución en la temperatura de los gases que llegan a la sección de baja presión, sin embargo, no tiene un efecto notable en el rendimiento como puede observarse en la figura 5. Se observa también que la variación del flujo másico es muy similar a la de la irradiancia, esto se debe a que, como se explicó anteriormente, las temperaturas de entrada y salida del economizador permanecen constantes y quien varía con respecto a la radiación es solo el flujo másico.

Por otro lado, también se realizó el análisis de la variación del costo de generación (gráfica con línea continua en la figura 5) y el rendimiento (gráfica con línea punteada en la figura 5) en función de la irradiancia directa. Se observó que se presenta una variación importante en estos dos parámetros, principalmente

En cuanto al número de bucles totales obtenidos en cada configuración, es importante aclarar que la diferencia es grande cuando se acopla el campo solar al economizador de alta, contrario a cuando se acopla al sobrecalentador de baja. Esta diferencia tan marcada radica en que el área de captación obtenida para el sobrecalentador de baja es muy pequeña porque este elemento demanda mucha menos energía que el economizador de alta presión. Mayor información acerca del tamaño de las superficies de intercambio de una CRC se puede encontrar en Durán et al. (2013).

Por otro lado, se realizó un análisis de sensibilidad en función de irradiancia directa, únicamente para la configuración B, que fue la que dio mejores resultados. Se observó que la irradiancia tiene una influencia muy importante sobre el flujo másico de agua en el canal parabólico, que al pasar por las secciones de la caldera se convierte en vapor. Esto se muestra en la gráfica de la figura 4. Esta tendencia se debe a que durante el análisis las temperaturas de entrada y salida de los economizadores permanecen constantes, entonces quien varía en función de la irradiancia es el flujo másico.

El análisis se realizó para las condiciones del mes de mayo, ya que es el que mejores condiciones de irradiancia presenta, se observó más de 6 horas que la irradiancia promedio es superior a la promedio considerada; en el rendimiento para valores de irradiancia entre 0.76 y $0.86 \mathrm{~kW} / \mathrm{m}^{2}$, intervalo en el cual el rendimiento cambia entre $55.6 \%$ y $57.2 \%$. Posteriormente, para valores de irradiancia menores a $0.76 \mathrm{~kW} / \mathrm{m}^{2}$, la turbina de gas compensa la falta de energía del campo solar y por esta razón la variación del rendimiento disminuye considerablemente. Por otro lado, se observa una pendiente constante en la variación del costo de generación con la irradiación, de hecho se obtuvo una correlación lineal con pendiente negativa que se presenta en la misma figura. De acuerdo con la gráfica y la ecuación obtenida

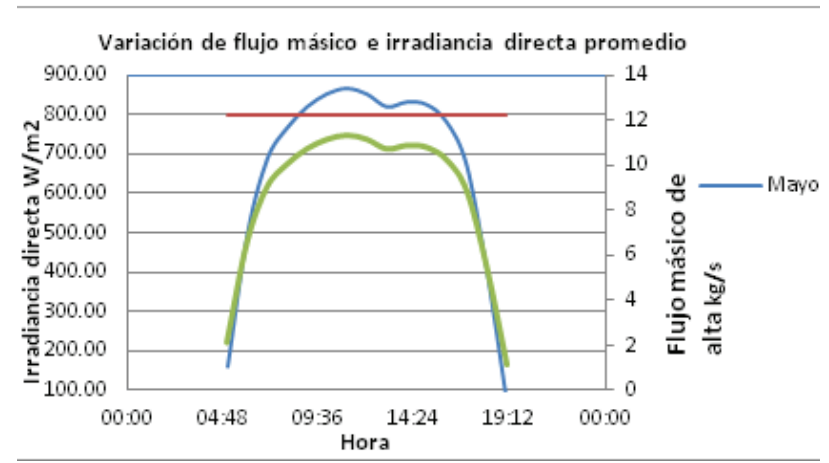

Figura 4. Variación del flujo másico de vapor en el canal parabólico de la configuración A como función de la irradiancia directa, graficado en función del tiempo horario 


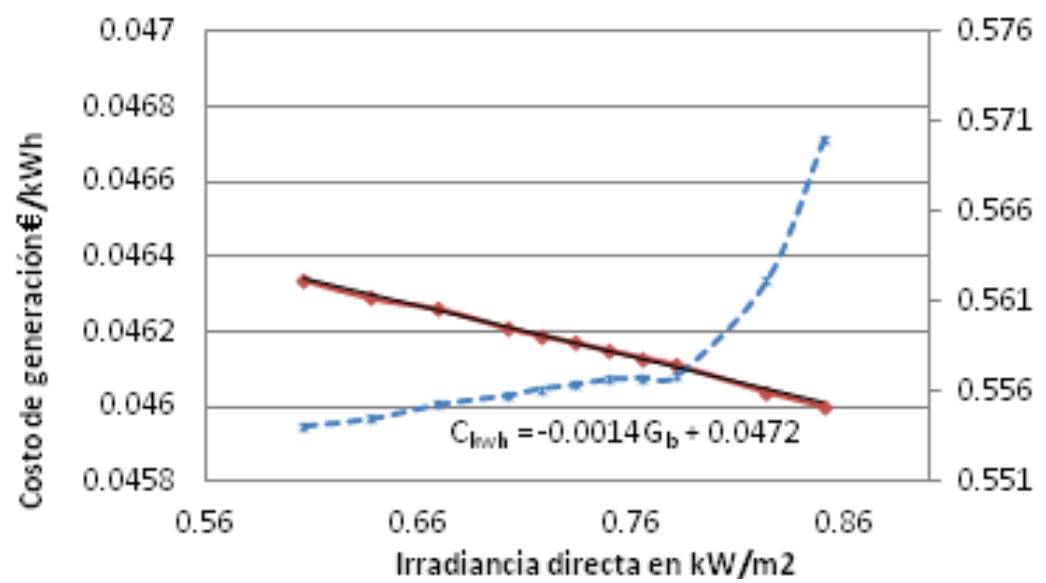

Figura 5. Variación del costo (línea roja) y rendimiento (línea azul) de la configuración A como función de la irradiancia directa se observa que aún con la disminución en el costo de generación, este permanece menor que el obtenido para la configuración de ciclo combinado sin la integración con el campo solar (tabla 7) aún para valores de irradiancia bajos.

\section{Conclusiones}

En este trabajo se aplicó un modelo de optimización termoeconómica con algoritmos genéticos para la optimización de diferentes configuraciones de ISCC. Se observó que la metodología es aplicable y brinda resultados interesantes.

Se aplicó el modelo a dos configuraciones de ISCC y se compararon los resultados con un CC convencional; se observa que la planta optimizada de ciclo combinado integrada con la planta solar (ISCC) presenta mejores resultados que la misma configuración de ciclo combinado optimizada, esto empleando el valor de irradiación directa promedio de la región de Cerro Prieto, B.C.

Asimismo, al considerarse la planta de canal parabólico como el economizador o el sobrecalentador de la CRC y tomando en cuenta un Approach Point mayor que $3^{\circ}$, no se espera tener evaporación en el absorbedor. Lo cual favorece el empleo de GDV y evita el uso de un aceite como fluido de trabajo.

Se observa que es preferible acoplar la planta de canal parabólico en el economizador de la sección de alta presión de la CRC; la razón es que de esta forma se tiene más energía disponible de los gases en la sección de baja presión de la misma y, por lo tanto, se puede incrementar el flujo másico en esta sección y la potencia del ciclo.

Por otro lado, se observó que la variación de la radiación directa tiene un efecto importante sobre el rendimiento y costo de generación; sin embargo, aún con esta variación este costo resulta menor que el obtenido con la configuración que no está integrada con el canal parabólico. Asimismo, resulta interesante resaltar que la gráfica de variación del costo de generación en función de la radiación, sigue una correlación lineal con pendiente negativa.

Se considera importante analizar otras configuraciones con y sin acoplamiento con la planta solar. Esto permitirá establecer tendencias en el diseño de este tipo de sistemas, además de que los diseñadores y tomadores de decisiones de empresas generadoras de energía tendrán una idea más clara de hacia dónde deben dirigirse los valores de los parámetros de diseño y qué desempeño y costo se espera tener, previo a seleccionar un diseño o adquirir una central llave en mano.

La GDV es una nueva opción que bajará los costos, ya que se eliminan el intercambiador de calor y los aceites sintéticos (fluido de transferencia de calor).

\section{Nomenclatura}

\begin{tabular}{lll}
\hline Símbolo & Significado & Unidad \\
\hline $\mathrm{A}_{\mathrm{A}}$ & Área de apertura del colector solar & $\mathrm{m}^{2}$ \\
$\mathrm{~A}$ & Área de transferencia de calor & $\mathrm{m}^{2}$ \\
$\mathrm{AP}$ & Approach point & $\mathrm{K}$ \\
$\mathrm{AP}$ & Alta presión & \\
$\mathrm{BP}$ & Baja presión & \\
$\mathrm{C}$ & Razón de concentración & \\
$\mathrm{C}$ & Costo & $\mathrm{M} €$ \\
$\mathrm{CC}$ & Ciclo combinado & \\
$\mathrm{C}_{\mathrm{p}}$ & Calor específico a presión constante & $\mathrm{kJ} / \mathrm{kg} \mathrm{K}$ \\
$\mathrm{C}_{\text {Tot }}$ & Costo Total & $\mathrm{M} €$ \\
$\mathrm{C}_{\mathrm{kwh}}$ & Costo de generación por $\mathrm{kWh}$ & $€ / \mathrm{kWh}$ \\
Cond & Condensador & \\
\hline
\end{tabular}




\begin{tabular}{|c|c|c|}
\hline CRC & Caldera de Recuperación de Calor & \\
\hline Desg & Desgasificador & \\
\hline DT & $\begin{array}{l}\text { Diferencia terminal de temperaturas en el so- } \\
\text { brecalentador }\end{array}$ & K \\
\hline EC & Economizador & \\
\hline EV & Evaporador & \\
\hline $\mathrm{F}_{\mathrm{R}}$ & Factor de remoción de calor & \\
\hline $\mathrm{F}^{\prime}$ & Factor de eficiencia del colector & \\
\hline $\mathrm{G}_{\mathrm{b}}$ & Irradiancia directa instantánea & $\mathrm{W} / \mathrm{m}^{2}$ \\
\hline GDV & Generación directa de Vapor & \\
\hline h & Horas de operación de la planta & $\mathrm{h}$ \\
\hline HRSG & Heat Recovery Steam Generator & \\
\hline ISCC & Integrated solar combined cycle & \\
\hline \multirow[t]{2}{*}{$\mathrm{k}$} & $\begin{array}{l}\text { Constate de costo correspondiente a una sec- } \\
\text { ción de la CRC }\end{array}$ & \\
\hline & Flujo másico & $\mathrm{kg} / \mathrm{s}$ \\
\hline PP & Pinch Point & $\mathrm{K}$ \\
\hline $\mathrm{Q}_{\mathrm{u}}$ & Calor útil & $\mathrm{W}$ \\
\hline SC & Sobrecalentador & \\
\hline $\mathrm{T}$ & Temperatura & K \\
\hline TV & Turbina de vapor & \\
\hline TG & Turbina de gas & \\
\hline $\mathrm{U}_{\mathrm{L}}$ & $\begin{array}{l}\text { Coeficiente global de transferencia de calor } \\
\text { del colector }\end{array}$ & $\mathrm{W} / \mathrm{m}^{2} \mathrm{~K}$ \\
\hline \multirow[t]{2}{*}{$W$} & Potencia & MW \\
\hline & Potencia media anual de la planta & MW \\
\hline$\alpha$ & Absortancia & \\
\hline Símbolo & Significado & Unidad \\
\hline$\eta$ & Eficiencia térmica & \\
\hline$\eta_{0}$ & Eficiencia óptica & \\
\hline \multirow[t]{2}{*}{$\tau$} & Transmitancia & \\
\hline & Subíndices & \\
\hline $\mathrm{i}$ & Interior & \\
\hline $\mathrm{a}$ & Ambiente & \\
\hline $\mathrm{AP}$ & Alta presión & \\
\hline BP & Baja presión & \\
\hline $\mathrm{sec}$ & $\begin{array}{l}\text { Sección de la caldera de recuperación } \\
\text { de calor }\end{array}$ & \\
\hline
\end{tabular}

\section{Referencias}

Almanza R., Estrada-Cajigal V. Irradiaciones global, directa y difusa, en superficies horizontales e inclinadas, así como irradiación directa normal en la República Mexicana. Serie de Investigación y Desarrollo, SID/646, Instituto de Ingeniería, UNAM, 2005.
Attala L., Facchini B. y Ferrara G. Thermoeconomic optimization method as design tool in gas-steam combined plant realization. Energy Conversion and Management, volumnen 42, 2001.

Baghernejad A. y Yaghoubi M. Exergoeconomic analysis and optimization of an integrated solar combined cycle system (ISCCS) using genetic algorithm. Energy Conversion and Management, volumen 52, 2011: 2193-2203.

Bakos G.C., Ioannidis I., Tsagas N.F., Seftelis I. Design optimization and conversion-efficiency determination of a line-focus parabolic-trough solar collector (PTC). Applied Energy, volumen 68, 2001: 43-50.

Duffie J.A. y Beckman W.A. Solar Engineering of Thermal Processes, $3^{\mathrm{a}}$ ed., John Wiley \& Sons, Inc, 2006.

Duran M. Estudio de calderas de recuperación de calor de ciclos combinados de turbinas de gas y vapor empleando la técnica de algoritmos genéticos, (tesis de doctorado), UPM, Madrid, España, 2004.

Durán M., Rovira A., Valdés M., Rincón E. A methodology for the

$\dot{m}$ geometric design of heat recovery steam generators applying genetic algorithms. Applied Thermal Engineering, volume 52 (número 1) 2013: 77-83.

Facchini S. y Stecco S. Cooled expansion in gas turbines: comparison of analysis methods. Energy Conversion and Management, volumen 40, 1999: 1207-1224.

GEF project Document, MEXICO: Hybrid Solar Thermal Power Plant Project, 2006 [en línea] [fecha de consulta: enero del 2013]. Disponible en: www.theGEF.org

Geyer M. et al. EUROTROUGH -Parabolic trough collector developed for cost efficient solar power generation, en: 11th Solar-

$\bar{W}$ PACES International Symposium on Concentrated Solar Power and Chemical Energy Technologies, Zurich, Switzerland, 2002

IEA-ETSAP y IRENA. Technology brief E10. 2013, [en línea] [fecha de consulta: enero del 2013]. Disponible en: www.irena.org/ Publications.

International Energy Agency (IEA). Solar Energy Perspectives. Renewable Energy Technologies ISBN: 978-9264124578, 2011 [en línea] [fecha de consulta: el 5 de julio del 2013]. Disponilbe en http://www.iea.org/

Kelhofer R.H. Combined-cycle gas and steam power plants, The Fairmont Press Inc., 1991, USA.

Lentz A. y Almanza R. Solar-geothermal hybrid system. Applied Thermal Engineering, volumen 26 (números 14-15), 2006: 15371544.

Llorente I., Álvarez J., Blanco D. Performance model for parabolic trough solar thermal power plants with thermal storage: Comparison to operating plant data. Solar Energy, volumen 85, 2011: 2443-2460.

Montes M. Análisis y propuestas de sistemas solares de alta exergía que emplean agua como fluido calorífero, (tesis de doctorado), E.T.S. de Ingenieros Industriales, Universidad Politécnica de Madrid, España, 2008. 
Montes M.J., Abánades A., Martínez-Val J.M. Performance of a direct steam generation solar thermal power plant for electricity production as a function of the solar multiple. Solar Energy, volumen 83, 2009: 679-689.

Montes M.J., Rovira A., Muñoz M., Martínez-Val J.M. Performance analysis of an Integrated Solar Combined Cycle using Direct Steam Generation in parabolic trough collectors. Applied Energy, volumen 88, 2011: 3228-3238.

Muñoz M., Valdés M., Muñoz-Dominguez M. Turbomáquinas térmicas: fundamentos del diseño termodinámico, Publication of the E.T.S. of Industrial Engineers (en español), Universidad Politécnica de Madrid, España, 2004.

Nezammahalleh H., Farhadi F.,Tanhaemami M. Conceptual design and techno-economic assessment of solar combined cycle system with DSG technology. Solar Energy, volumen 84, 2010: 1696-1705.

Rovira A. Desarrollo de un modelo para la caracterización termoeconómica de ciclos combinados de turbinas de gas y de vapor en condiciones de carga variable, (tesis de doctorado), UPM, Madrid, España, 2004.

Tiwari G. Solar energy, fundamentals, design, modelling and Applications, Ed. Alpha Science, India, 2002.

Toffolo A. y Lazzareto A. Evolutionary algorithms for multi-objective energetic and economic optimization in thermal system design. Energy, volumen 27, 2001: 549-567.

Tsatsaronis G. y Pisa J. Exergoeconomic evaluation and optimization of energy systems application to the CGAM problem. Energy, volumen 19 (número 3), 1994: 287-321.
Tyagi S.K., Shengwei W., Singhal M.K., Kaushik S.C., Parkd S.R. Exergy analysis and parametric Study of concentrating type solar collectors. International Journal of Thermal Sciences, volumen 46 (número 12), 2007: 1304-1310.

U.S. Department of Energy. 2010 Solar Technologies Market Report, Energy Efficiency \& Renewable Energy, 2011.

Valdés M., Durán M.D., Rovira A. Thermoecomic optimization of Combined Cycle Gas Turbine Using Genetic Algorithms. Applied hermal Engineering, volumen 23 (número 17), 2003: 2169-2182.

Valdés M., Rovira A., Fernández J.A. The influence of atmospheric conditions on the performance of combined cycle gas turbine power plants, Proceedings of GT2006 ASME Turbo Expo 2006: Power for Land, Sea and Air, Barcelona, Spain, 2006a.

Valdés M., Rovira A., Durán M. On existence of trends applicable to thermoeconomic optimisation of combined cycle gas turbine power plants, Int. Journal of the Energy Insitute, volumen 6, (número 2), 2006b.

Valero A., Lozano M., Serra L., Torres C. Application of the exergetic cost to the CGAM Problem. Energy, volumen 19 (número 3), 1994: 365-381.

Wagner W. y Kretzschmar H. International Steam tables, properties of water and steam based on the industrial formulation, IAPWS-IF97, Springer, 1988.

Zarza E., Rojas M.E., González L., Caballero J.M., Rueda F. INDITEP: The first pre-commercial DSG solar power plant. Solar Energy, volumen 80, (número10), 2006: 1270-1276. 


\section{Este artículo se cita:}

Citación estilo Chicago

Durán-García, María Dolores, Rafael Almanza-Salgado, Ivan Galileo Martínez-Cienfuegos. Estudio del acoplamiento de ciclo combinado con planta solar de canal parabólico. Ingeniería Investigación y Tecnología, XVI, 02 (2015): 253-263.

\section{Citación estilo ISO 690}

Durán-García M.D., Almanza-Salgado R., Martínez-Cienfuegos I.G. Estudio del acoplamiento de ciclo combinado con planta solar de canal parabólico. Ingeniería Investigación y Tecnología, volumen XVI (número 2), abril-junio 2015: 253-263.

\section{Semblanzas de los autores}

María Dolores Durán-García. Profesor-investigador de tiempo completo en la Facultad de Ingeniería de la Universidad Autónoma del Estado de México. Es ingeniera mecánica de profesión con maestría en técnicas de energías renovables y doctorado en ingeniería térmica. Ha realizado proyectos relacionados con la optimización termoeconómica de sistemas térmicos y plantas de potencia. Asimismo ha realizado proyectos y escrito artículos referentes a concentración solar, principalmente, plantas de canal parabólico.

Rafael Almanza-Salgado. Investigador titular C en el Instituto de Ingeniaría, UNAM, ha trabajado en proyectos de energía solar por más de 38 años. Tiene nivel 3 en el Sistema Nacional de Investigadores y obtuvo el Premio Universidad Nacional 2006, Innovación Tecnológica y Desarrollo Industrial.

Iván Galileo Martínez-Cienfuegos. Profesor-investigador de tiempo completo en la Facultad de Ingeniería de la Universidad Autónoma del Estado de México. Ingeniero químico de carrera con maestría en ingeniería en energía orientada a procesos y uso eficiente de la energía. Doctor en ingeniería en energía, con el proyecto de investigación de flujo bifásico de agua en sistemas termosolares de canal parabólico para generación de electricidad. Ha realizado otros proyectos en la línea de integración de sistemas termosolares y fotovoltaicos para desalación de agua, así como geoquímica del agua de mar en descargas de plantas desaladoras. Sus líneas de investigación son: generación directa de vapor para sistemas termosolares, materiales para concentración solar y modelos de radiación solar. 